\title{
ECOTOX, the INRA's network of ecotoxicologists, a major structure involved for the coordination and structuring of the French research in ecotoxicology
}

\author{
Christian Mougin $^{1} \cdot$ Agnès Bouchez $^{2} \cdot$ Laurence Denaix $^{3} \cdot$ François Laurent $^{4}$. \\ Fabrice Martin-Laurent ${ }^{5}$
}

Received: 2 December 2015 / Accepted: 7 December 2015 /Published online: 22 December 2015

(C) Springer-Verlag Berlin Heidelberg 2015

A great number of chemicals (metallic and synthetic compounds) are used by human activities. Most of them enter our environment and lead to its contamination, as well as to adverse effects on wildlife and humans. Some compounds of natural origin (toxins, biopesticides...) are also of concern. One challenge for ecotoxicologists remains to understand and predict the long-term impact of these mixtures of chemicals, often on trace concentrations, on living organisms. This implies to develop tools and assessment strategies at different biological levels: individuals, populations, and communities.

In that context, this special issue of Environmental Science and Pollution Research highlights selected papers from the members of ECOTOX, the network of ecotoxicologists of the National Institute for Agricultural Research (INRA) (http://www6.inra.fr/ecotox_eng/). Set up in 2009 to

Responsible editor: Philippe Garrigues

Christian Mougin

christian.mougin@versailles.inra.fr

1 INRA, UMR1402 ECOSYS (Joint Research Unit for the Functional Ecology and Ecotoxicology of Agro-Ecosystems), F-78026 Versailles cedex, France

2 INRA, UMR0042 CARRTEL (Alpine Research Centre for Food Webs in Lentic Ecosystems), F-74203 Thonon-les-Bains cedex, France

3 INRA, UMR1391 ISPA (Joint Research Unit for Soil-Plant-Atmosphere Interactions), F-33883 Villenave d'Ornon cedex, France

4 INRA, UMR5245 ECOLAB (Laboratory of Functional Ecology), F-31326 Castanet Tolosan cedex, France

5 INRA, UMR1347 (Joint Research Unit for Agroecology), F-21065 Dijon, France stimulate research on ecotoxicology within INRA and to unify the teams involved in the field, the network now brought together academics of other French research institutes and universities, as well as professionals. A total of 150 scientists over 50 laboratories or companies subscribes to the mailing list of the network. The ECOTOX network is sponsored by the INRA, and particularly the scientific divisions "Forest, Grassland and Freshwater Ecology" and "Environment and Agronomy", as well as the "Sustainable Management of Crop Health" metaprogram $(\mathrm{SMaCH})$.

The network has three main priority objectives: (i) to encourage scientific thinking within the community of ecotoxicologists, whether they are dealing with aquatic or terrestrial ecosystems, (ii) to contribute to defining a national research strategy in this field, and (iii) to favor scientific production. For many years, the network offers opportunities for exchanges within the French community of ecotoxicologists, especially through regular scientific seminars They were held in Thonon les Bains (1999), Antibes (2002), Dinard (2006), St-Lager (2011), and Biarritz (2014); see the conference report on the last seminar in the present special issue. The network is also involved in the coordination and structuring of the national research in ecotoxicology, by interacting with other French networks and structures. In addition, the network has also set up a thematic watch system (http://www6.inra.fr/ecotox_eng/ Watch), providing to numerous partners a bimonthly bulletin. Finally, the network provides means to present and publish the results and highlights from the research developed by its scientists. That special issue is one illustration of that objective.

Twenty-three papers were included in this special issue. These papers highlighted current research on the fate, mode of action, and adverse outcomes for a variety of environmental contaminants, on a variety of biological targets. Some of these results were presented during the last seminar in Biarritz. They aimed at establishing the 
relationships between environmental factors, toxic chemicals, and their effects on eukaryotic organisms.

The first papers concerned the assessment of the bioavailability of chemicals to invertebrates and plants. In that context, a mild extraction method was proposed by Nélieu et al. to study the bioavailability of the fungicide epoxiconazole to the earthworm Aporrectodea icterica. The amounts of epoxiconazole extracted by an hydroxypropyl- $\beta$ cyclodextrin solution were identical to these determined in A. icterica exposed in real spiked soil. This mild method was sensitive to soil sorption capacity and to aging. The study could be expanded to other organic contaminants in order to tend towards a reliable predictability of the ecotoxicological risks which would take bioavailability in consideration.

Pauget et al. were interested in determining the environmental parameters influencing the metal bioavailability and transfer to snails exposed to multiple contamination sources. They showed the great contribution of lettuce in Cd assimilation by snails, reinforcing the fact that measuring only the total soil concentration was not sufficient to accurately assess $\mathrm{Cd}$ bioavailability. They concluded that improving biodynamic model data on sources contribution under a complex exposure remains necessary to assess metal bioavailability to snails for risk assessment purpose.

Predicting the soil-to-plant transfer of metals in the context of global warming has become a major issue for food safety. It requires a better understanding of how the temperature alters the bioavailability of metals in cultivated soils, as studied by Cornu et al. The soil-to-plant transfer of $\mathrm{Cd}$ and $\mathrm{Zn}$ was promoted at higher soil temperatures despite a parallel decrease in $\mathrm{Cd}$ and $\mathrm{Zn}$ bioavailability. Warming is thus suspected to promote the soil-to-plant transfer of metals by facilitating the capture of metals by plant roots and, in agricultural soils, may lead to an over concentration of metals like $\mathrm{Cd}$ in the edible parts of crops.

Then, the relevance of innovative processes and exogenic materials in modifying the exposome of fauna and plants to ensure appropriate remedial solutions of polluted soils was assessed. Mixed effluents of urban and hospital effluents were tested by Wigh et al. before treatment, after biological treatment alone, and after biological treatment followed by a tertiary ozonation. Contrary to the lack of toxicity observed with normalized ecotoxicity tests, endpoints measured on zebrafish embryos such as mortality, developmental abnormalities, and genotoxicity demonstrated a residual toxicity of wastewaters after biological treatment, followed or not by ozonation. This work highlighted the long way to dispose of a valuable ecotoxicity bioassay battery adapted to the peculiarities of treated wastewater effluents.

In a similar way, the eco-genotoxicity of sludge was assessed in a laboratory soil test before their use for remediation in real conditions (Chiochetta et al.). They have shown that biotests (microbiological enzymes, earthworms, and higher plants including Vicia faba genotoxicity test) help to determine the most appropriate higher plants for this later purpose and to ascertain the application rates of sludge to promote soil revegetation and ecological restoration of a site impacted by anthropic activities.

Finally, Rorat et al. used specific biomarkers to assess the influence of exposure to sewage sludge on the composting earthworms Eisenia fetida and Eisenia Andrei, also recommended by OECD as test organisms in ecotoxicology. Considering specific biomarkers, they showed that contaminants present in sewage sludge did not affect earthworm's immune system, but the mechanisms of accumulation and detoxification of particular metal fractions by earthworms should be explained.

Research was also designed to assess the impact of chemical stressors encountered in agro-ecosystems (organic contaminants, trace elements, and biological control agents). They concerned different model organisms of aquatic and terrestrial ecosystems.

Firstly, Lebrun et al. conformed that soil fungi of different phyla-secreted hydrolases and oxidoreductases involved in various environmental processes. They demonstrated that, unlike the great variability of the response of hydrolases, the secretion of oxidoreductases was increased or specifically produced following exposure of fungi to $\mathrm{Cu}$ and $\mathrm{Cd}$. Because of the genericity of these responses, the fungal oxidoreductases appear relevant tools for metal ecotoxicity assessment in soils.

The choice of incoming data for building Species Sensitivity Distributions (SSD) plays a crucial role and may change greatly the values of the hazardous concentrations thresholds. In their study considering planktonic species, Larras et al. showed that SSD based on literature data were more sensitive than the one obtained with specific data. They conclude that in SSD models based on data that underestimate the sensitivity of species, the hazardous concentration 5 (that affect $5 \%$ of the species of a community) thresholds less protective than expected. Additional studies on communities are important to increase the predictive power of the threshold defined for risk assessment.

The effects of pollutants were also assessed on invertebrates. Pelosi et al. showed that the commercial formulation of the fungicide epoxiconazole (Opus ${ }^{\circledR}$ ) exerted more negative effects on the earthworm A. icterica than this observed on Eisenia fetida., a species commonly used in assays for ecotoxicity assessment. They highlighted the importance of considering exposure duration and kinetic measurements when studying biochemical biomarkers.

Developmental stage was shown to govern snail sensitivity to $\mathrm{Cd}$ by Baurand et al. They evidenced differential sensitivities in early the life stages of Cd-exposed terrestrial snail embryos. In particular, these age-dependent differences reflected the variability of the individual hatching success and varying expression levels of the Ca-CdMT gene in 
response to $\mathrm{Cd}$ exposure. Cd sensitivity was found to decrease with the age and developmental stage of Cantareus aspersus embryos at the time of $\mathrm{Cd}$ exposure. In a second study, they showed that long-term exposure to $\mathrm{Cd}$ and $\mathrm{Cu}$ in developing snail embryos can lead to the expression of all three snailspecific MT isoform genes. The investigation shows that the kind of metal exposure (duration of exposure, concentration levels of metals involved) may have a strong impact on the upregulation pattern of the three-snail MT genes.

Then, the effects of low doses of insecticides were assessed on both targeted crop pests and beneficial insects. These doses could induce unexpected hormetic effects on insects. Siaussat et al. aimed at examining the effects of sublethal doses of deltamethrin on the peripheral olfactory system and sexual behavior of the cotton leafworm Spodoptera littoralis. They highlighted a hormetic effect of sublethal dose of deltamethrin on the male responses to sex pheromone, without any modification of their response to host-plant odorants. That effect can potentially contribute to the adaptation of this pest insect to pesticide treatment. They studied also the effects of sublethal doses of methomyl and chlorpyrifos on S. littoralis. A metabolomic approach developed by Dewer et al. evidenced that these insecticides had a systemic effect on the treated insects. The metabolic disruption and the info-disruption of the chemical communication they reported participated to a better understanding of the subtle effects of environmentally relevant sublethal pesticide concentrations on a target species.

Moreover, beside conventional organic pesticides, biological control agents are used for crop protection. Amichot et al. evidenced, as side effect, an acute toxicity of Bacillus thuringiensis on Trichogramma chilonis females. After ingestion of $B$. thuringiensis at sublethal doses, the life history traits of $T$. chilonis such were differently affected. These effects, taken together, exemplify the difficulty to address ecological risks of an active compound.

Several studies were concerned with higher plants. Marchand et al. studied the foliar ionome, photosystem II activity, and leaf growth parameters of Ranunculus acris $L$., a potential biomonitor of trace element contamination and phytoavailability in two riverbank soils. They showed that the foliar ionome of $R$. acris was only effective to biomonitor total and soluble soil $\mathrm{Na}$ and Mo concentrations in soil series. In a second study, they considered the plant responses to a phytomanaged urban technosol contaminated by trace elements and polycyclic aromatic hydrocarbons. They showed as necessary to characterize the whole pollutant linkage when estimating the risks associated to high trace elements/PAH concentrations in a soil. Because the phytotoxicity may be, as in their study, relatively low in spite of high total contaminant concentrations, the implementation of phytomanagement strategies will limit in a long-lasting way the solubilization and/or mobility of contaminants at lower cost than the techniques of civil engineering used for soil decontamination.

Understanding the mechanisms developed by organisms to tolerate pollutants remains of great concern. Bernard et al. analyzed the expression of antioxidant defense genes in Brassica oleracea and Trifolium repens. Plants exposed to suburban soil spiked by low concentrations of $\mathrm{Cd}$ and $\mathrm{Pb}$ were analyzed for metal accumulations, expression levels of genes encoding proteins involved, and/or relatedness to antioxidant defense systems. The results presented suggested a benefit to use complementary species to better apprehend the biological effects in ecotoxicology, because distinct species do not mobilize exactly the same mechanisms to manage with metal contamination. This must be kept in mind when gene variations and in a larger extent biomarkers are used to assess soil quality.

Several papers were also concerned with the transfer of contamination within feed or food chains in a context of agricultural practices. The work presented by Nguyen et al. considered the exposure of maize plant to low $\mathrm{Cd}$ concentrations. The $\mathrm{Cd}, \mathrm{Cu}$, and $\mathrm{Zn}$ concentrations in shoots decreased with time at the vegetative stages. The shoots of young plants were enriched in micronutrients $\mathrm{Cu}$ and $\mathrm{Zn}$ but also in toxic $\mathrm{Cd}$, illustrating the challenge to consider plants containing both nutritional value and levels of toxic elements. The xylem sap analysis is a potential valuable tool to precociously compare different soil conditions with respect to both the phytoavailability of the trace elements and their allocation to shoots.

The high tolerance of the herbivorous snail C. aspersus to $\mathrm{Hg}$ when exposed through food or soil contamination was demonstrated by Gimbert et al. The ecotoxicological metrics provided by their study will contribute to the databases for $\mathrm{Hg}$, remaining still sparse concerning the terrestrial environment. Their results made snails of interest for the biomonitoring and thus, as they may represent a non-negligible source of $\mathrm{Hg}$ transfer to their consumers, for the risk assessment of $\mathrm{Hg}$ contaminated sites.

Livestock reared on polluted areas are involuntarily contaminated by chemicals. Their level of contamination may exceed the threshold values set by the European Union. In their study, Lastel et al. aimed at characterizing chlordecone disappearance in the empty carcass, the liver, the adipose tissues, and the serum in growing ruminants. Their suggestion that sustainability of farming and rearing practices used in the French West Indies remained possible when the contaminated animals underwent a sufficient period of decontamination. That period of decontamination needs to be adjusted to each kind of animals and their initial level of contamination.

The study of crossed-effects between chemical pollution and climate change was identified as an increasing relevant concern for ecotoxicologists, by possibly modifying community structure and ecosystem functioning. Gandar et al. 
assessed the individual and crossed-effects of pesticide exposure and temperature rising on goldfish, Carassius auratus. They found that combined chemical and thermal stresses impaired the capacity of the fish to set up an efficient adaptive response, and suggested that temperature will make fish more sensitive to water contamination by pesticides. These results raised concerns about wild fish conservation submitted to global changes.

To conclude that special issue, we mention the infrastructure for Analysis and Experimentation on Ecosystems (AnaEE-France), an integrated network of the major French experimental, analytical, and modeling platforms dedicated to the biological study of continental ecosystems (both aquatic and terrestrial). AnaEE-France offers adequate services to tackle the new challenges of research in ecotoxicology, positioning its various types of platforms in an ecologically advanced ecotoxicology approach (Mougin et al., 2015, Environ Sci Pollut Res 22:16215-16228).

We hope that you will enjoy reading this special issue, presenting some challenging questions in ecotoxicology, from bioavailability of pollutants to remediation and ecological engineering.

Acknowledgments The network extends its sincere thanks to the Environmental Science and Pollution Research editor-in-chief Prof. Philippe Garrigues and to Springer, the journal's publisher, for their full support to that special issue. We are also grateful to the ESPR Editorial Board and reviewers for undertaking careful review of manuscripts submitted to this special issue.



Christian Mougin is a senior scientist at the National Institute for Agricultural Research (INRA) in the "Functional Ecology and Ecotoxicology of Agro-Ecosystems" joint research unit in Versailles, France. His main interests combine the fate of contaminants (metals, pesticides, cyanotoxins, and emerging compounds) in soils and the responses of microorganisms and macrofauna exposed to these chemicals, both in situ and through laboratory experiments. His work focuses actually on the development of innovative tools for the biochemical characterizations of soils, sediments, and living micro-macroorganisms, in relation to the assessment of ecosystems functioning under anthropic constraints. Christian Mougin is in charge of French research platforms open to research in ecotoxicology and leads the INRA's national network of Ecotoxicologists, ECOTOX (http://www6.inra.fr/ecotox_eng/).

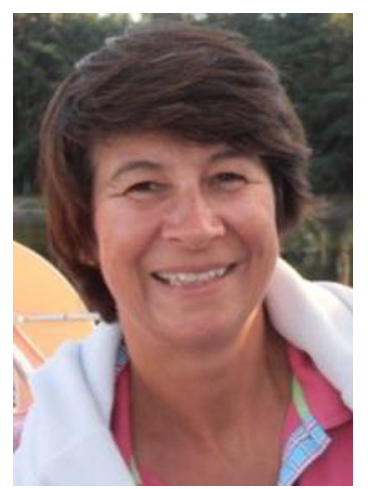

Agnès Bouchez is a senior scientist at the National Institute for Agricultural Research (INRA) in the "Alpine Center for Research on Lake Ecosystems and Food Webs" research unit in Thononles-bains, France. She is mainly involved in the functional evaluation of the ecological quality of lakes and rivers using genomic, taxonomic, and ecotoxicological tools. Based on these tools, she develops innovative metrics and methodologies to assess the impacts of xenobiotics on the structure and functioning of freshwater microbial communities. Her work focused on bringing more ecological realism in risk or impact assessment, regarding contaminants (low doses, mixtures), biological organisms (species or communities from the environment), and experimental approaches in micro-mesocosms or in the field. Agnès Bouchez is co-leading the INRA's national network of Ecotoxicologists, ECOTOX (http://www6. inra.fr/ecotox_eng/).

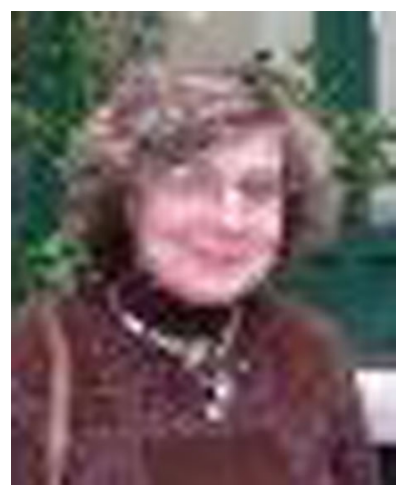

Laurence Denaix is a senior scientist at the National Institute for Agricultural Research (INRA) in charge of the direction of the research unit "Interactions of SoilPlant Atmosphere" in Bordeaux, France. Her main research topics are the bioavailability of trace elements, their speciation in soil solution, and the quantification of their soil-root flows, using passive samplers. She is working on diffuse contamination of agroecosystems and also manages transfer projects with technical institutes and participates to the management of the RMT QUASAPROVE. She teaches the terrestrial ecotoxicology at Bordeaux University, in the master "Ecotoxicology and environmental chemistry" and is a member of the INRA's national network of Ecotoxicologists, ECOTOX. Laurence Denaix is co-leading the INRA's national network of Ecotoxicologists, ECOTOX (http://www6.inra.fr/ecotox_eng/). 


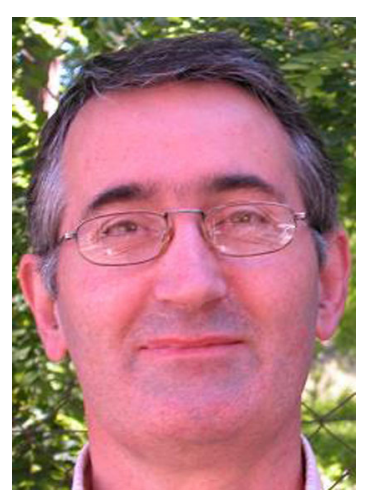

François Laurent is senior scientist at the National Institute for Agricultural Research (INRA) in the "Research Centre in Food Toxicology" in Toulouse. He is concerned with the role of plants in chemical (pesticides, POPs, emerging compounds) exposures of food chains, by the study of the fate of these compounds in plants. Currently, he develops a new research theme on the variability of the response of macrophytes to the chemical stress. In parallel, he studies plant potential for ecosystem services in the remediation of chemical-polluted sites. F. Laurent is secretary of the Scientific Commission of Phytopharma Prize (http://www.gfpesticides.org/prix-phytopharma.html).

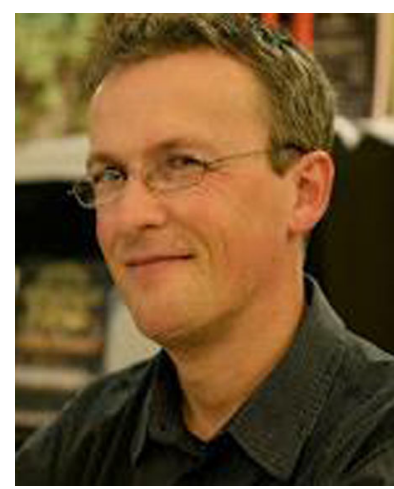

Fabrice Martin-Laurent is a senior scientist at the National Institute for Agricultural Research (INRA) in the Agroecology research unit in Dijon, France. He is leading a research team studying microbial ecology of agricultural chemicals including both fertilizers and pesticides. His main interest is focused on the pesticides microbial ecotoxicology. On one hand, his work is aiming at developing innovative approaches based on molecular approaches to assess the impact of these chemicals on the composition, abundance, diversity, and activity of soil microbial community. On the other hand, his is studying the processes responsible for the selection in the soil environment of microorganisms able to rapidly biodegrade pesticides. Fabrice Martin-Laurent is co-leading the INRA's national network of Ecotoxicologists, ECOTOX (http://www6.inra.fr/ecotox_eng/), is the co-creator of the French Network of Microbial Ecotoxicology (http://www.ecotoxicomic.fr/), and is member of the managing committee of the RTP of Microbial Ecotoxicology supported by INEE and INSU of CNRS. 